\title{
Pharmacotherapy prescribing patterns in the treatment of bipolar disorder in a South African outpatient population
}

\author{
'Department of Psychiatry, Faculty of Health Sciences, University of the Witwaters- \\ rand, 7 York Road, Parktown, 2193, Johannesburg, South Africa \\ *email: Eleanorholzamweb.co.za; Christopher.szabolawits.ac.za \\ DOI: 10.2478/gp-2018-0006 \\ Received: 8 March 2018; Accepted: 30 May 2018
}

\begin{abstract}
Objectives: To describe the range and frequency of psychotropics used in the management of bipolar disorder in a specific setting as well as describe the nature and frequency of monotherapy versus polypharmacy use. Specifically to determine congruence with recommended standard of care that might inform a review and refinement of prescribing within this context.

Methods: The study was a retrospective file review for patients with bipolar disorder (ICD 10 and or DSM IV TR diagnostic criteria) attending the outpatient clinic of a specialised psychiatric hospital (Tara Hospital) affiliated to the Department of Psychiatry (University of the Witwatersrand) in Johannesburg, South Africa. Data were presented both descriptively (continuous, discrete and categorical data) and with a range of appropriate statistical tests used for associations between categorical data (Chi Square; Fisher's exact test; Cramer's $V$ and the phi coefficient).

Results: The majority of patients (93.8\%) were prescribed polypharmacy, with 3.2 as the mean number of psychotropic medications prescribed per patient (as determined from the last prescription written during the period of review, i.e. one year). There was a notable variety in the combinations used with no particular combination being prescribed in the majority of patients, noting, however, that $47 \%$ of the combinations used included a standard mood stabiliser prescribed together with an antipsychotic.

Conclusions: The current study provides data on the prescribing patterns for bipolar disorder in a specialist psychiatric clinic within an academic complex in South Africa. The findings reflect international studies and highlight polypharmacy and combination treatment as common in such settings. Where polypharmacy is prescribed, the variation in combinations prescribed indicate that whilst treatment guidelines may provide a best practice approach, studies determining the most useful combinations are few and ultimately clinical requirements for individual patients will dictate treatment.
\end{abstract}

\section{Keywords}

Drug therapy, bipolar disorder, outpatient, South Africa.

\section{INTRODUCTION}

Bipolar disorder is a mood disorder characterised by manic, hypomanic and depressive episodes (Frye 2011). Pharmacological treatment is crucial. Such treatment is aimed for acute exacerbation of a manic, hypomanic or depressive episode, preventing relapses and recurrences and improving inter-episode functioning (remission/recovery) (Jarema et al. 2005).

The medications used to treat this disorder include mood stabilisers (lithium and anticonvulsants), antipsychotics (typical and atypical), antidepressants, hypnotics and anxiolytics (Jarema et al. 2005). These medications may be prescribed on their own or as polypharmacy and used in various combinations (Jarema et al. 2005). Polypharmacy refers to the use of two or more psychiatric medications, the total count of psychotropics prescribed for a patient (total drug load) (Frye et al. 2006; Goodwin et al. 2009; Kukreja, Kalra Shah \& Shrivastava 2013; Goldberg et al 2009). Complex polypharmacy refers to the use of four or more psychiatric medications (Frye et al. 2006; Goodwin et al. 2009; Kukreja, Kalra Shah \& Shrivastava 2013; Goldberg et al. 2009). The term combination treatment includes all the ways in which one psychotropic may be added to another in polypharmacy use (Frye et al. 2006; Goodwin et al. 2009; Kukreja, Kalra Shah \& Shrivastava 2013; Goldberg et al. 2009). These combinations include co-use, add on, augmentation and adjunctive prescribing (Goodwin et al. 2009; Kukreja, Kalra Shah \& Shrivastava 2013).

There are many clinical reasons why polypharmacy is used to treat bipolar disorder ranging from comorbid psychiatric conditions to partial response or failure of response to 
monotherapy and management of side effects (Goodwin et al. 2009; Kukreja, Kalra Shah \& Shrivastava 2013; Tohen et al. 2002; Post 2004). Whilst the evidence for polypharmacy and combination treatment is limited, the use in clinical practice is common (Solomon et al. 1996), noting that not all patients with bipolar disorder can be stabilised with monotherapy and that polypharmacy and combination treatment is increasingly used to manage these patients in clinical practice (Goldberg et al. 2009; Tohen et al. 2002; Post 2004; Solomon et al. 1996; Gever 2010; Ventimiglia, Kalali \& McIntyre 2009; Baldessarini et al. 2007; Post et al. 2010; Hooshmand et al. 2014). Clinicians, however, need to be mindful that the use of polypharmacy and combination treatment is therapeutic and rational and where possible evidence based (Kukreja, Kalra Shah \& Shrivastava 2013).

Patients with bipolar disorder managed in a specialist psychiatric setting have a greater chance of being managed with polypharmacy than in a general practice setting (Ventimiglia, Kalali \& McIntyre 2009; Nichol, Stimmel \& Lange 1995). The STEP BD study (1999-2005), further demonstrated that patients being treated at tertiary academic centres were more likely to be prescribed polypharmacy (Goldberg et al. 2009). Interestingly, a more recent Taiwanese study found that polypharmacy was less likely following admission to a medical centre ( $>500$ beds) (Hung et al., 2014).

International treatment guidelines also indicate that single drug or multiple drug therapy is acceptable based on the severity of the illness (Fountoulakis et al. 2005, 2007). There is, however, great debate in the literature as to whether clinical trials supporting the use of polypharmacy and combination treatment in all phases of bipolar disorder are valid and of high enough standard to provide adequate proof for evidenced-based clinical practice and use in treatment guidelines (Solomon et al. 1996; Alda \& Yatham 2009).

The current study aimed to investigate clinician prescribing patterns for adult patients with bipolar disorder attending an outpatient clinic at a specialist psychiatric hospital within an academic complex in South Africa.

It was hypothesised that within the study setting,

1. Polypharmacy would be more common than monotherapy

2. Polypharmacy would predominantly comprise at least two agents.

3. The agents used in polypharmacy would predominantly comprise a mood stabiliser and an antipsychotic.
The study objectives were thus

1. To describe the type and frequency of psychotropics used in the management of bipolar disorder.

2. To describe the nature and frequency of monotherapy versus polypharmacy use in bipolar disorder management, which includes the following classes and sub classes of psychotropics: lithium, standard anticonvulsants (sodium valproate, carbamazepine), novel anticonvulsants (lamotrigine, gabapentin, topiramate, oxcarbazepine), antipsychotics (typical, atypical), antidepressants (selective serotonin reuptake inhibitors [SSRIs], serotonin noradrenalin reuptake inhibitors [SNRIs], noradrenalin reuptake inhibitors [NRIs], tricyclic antidepressants [TCAs], mono-amine oxidase inhibitors [MAO's] and other antidepressants) and add-ons (benzodiazepines, non-benzodiazepines). The medications were classified for the purposes of the study as class and subclass in order to provide a structure for capturing a range of agents that required differentiation, that is, the anticonvulsants, the antipsychotics as well as the antidepressants.

Given the aforementioned, the significance of the study relates to

1. Providing specific data that would enable comparison with standard of care - as per existing guidelines.

2. Providing such data within a specific context.

3. Informing clinical practice based on the findings, that is, with review/refinement if required.

\section{MATERIAL AND METHODS}

A retrospective patient file review was undertaken.

\section{Setting}

The clinical files reviewed were those of adult (18 years old and above) patients attending the Tara Hospital psychiatric outpatient clinic. Tara Hospital outpatient clinic is located in Johannesburg, South Africa, and forms part of the University of the Witwatersrand's Department of Psychiatry's academic complex. The clinic provides secondary, tertiary and quaternary specialist psychiatric care.

\section{Sample}

The files of every patient who attended the clinic at least once in 2009 (from January to December) were screened (the year in question was selected in relation to the author undertaking 
research for the completion of her Master of Medicine [MMed] degree in 2009). The screening of the files from 2009 began in January 2013 and was completed in December 2013, that is, there was a delay between year selection and commencement of review (based on personal reasons).

This current study was based on the application of diagnostic criteria and principles of the International Classification of Diseases version 10 (ICD 10) (World Health Organization, 1992) and the Diagnostic and Statistical Manual of Mental Disorders version IV TR (DSM IV TR) (American Psychiatric Association, 2000). The DSM diagnoses bipolar subtypes, which include bipolar I (at least one manic episode); bipolar II (no mania, at least one hypomanic episode and one depressive episode) and bipolar not otherwise specified [NOS] (bipolar mood symptoms but not meeting criteria or any specific bipolar disorder) (American Psychiatric Association, 2000). It should be noted that in South Africa, ICD is used for coding all patient diagnoses including those with psychiatric disorders for the purposes of Government data capturing and medical scheme reimbursements. DSM is used clinically by mental health professionals; hence, both classification systems are operational in the South African psychiatric setting.

The files included in the study were those files that recorded the ICD 10 code (World Health Organization, 1992) corresponded with a bipolar disorder diagnosis (F31.0-F31.9) or single manic episode (F30.1, F30.2). Where the recording of the ICD 10 code (World Health Organization, 1992) was missing or incomplete, further scrutiny of the clinical notes enabled the researcher to establish a diagnosis of bipolar disorder using the ICD 10 coding criteria (World Health Organization, 1992) and/or DSM IV TR diagnostic criteria (American Psychiatric Association, 2000) and, therefore, include the patient file in the study. Files that did not meet the diagnostic/coding criteria for bipolar disorder or in which the clinical information was insufficient to allow the researcher to adequately diagnose/code bipolar disorder were excluded.

\section{Data}

Aside from demographic data (age, gender, socio-economic status as defined by hospital classification), patients in the sample were classified into the bipolar subtypes (I, II and NOS) according to DSM IV TR diagnostic criteria (American Psychiatric Association, 2000) and evidence of psychiatric comorbidity (yes or no) by referring to axis I and axis II conditions according to DSM IV TR (American Psychiatric Association, 2000) was recorded.
Patients in the sample were included irrespective of the number of visits they attended at the clinic from January to December 2009. Data regarding the psychotropic medication prescribed for each patient in the sample was obtained from the last prescription written by the doctor for the year 2009.

The following information was obtained from the relevant prescription:

1. Total number of psychotropics prescribed for the treatment of bipolar disorder.

2. Psychotropics prescribed for the treatment of bipolar disorder including class and subclass of medication.

\section{Statistical methods}

The information obtained from the file was sufficient to achieve the primary aim of the study.

\section{Sample Size}

The key research question was regarded as the proportion of patients treated with polypharmacy. This was measured as a proportion. This proportion was estimated to be $55 \%$ to $60 \%$ based on international literature (Tohen et al. 2002; Post, 2004; Gever, 2010; Post et al. 2010). For sample-size estimation, 55\% was used as a worst-case estimate. To estimate this proportion with $5 \%$ precision required a sample size of 381 , based on a sample size calculation for the estimation of proportions. The sample size of 242 used in the study was considerably lower and thus led to the estimation of a $55 \%$ proportion with only an approximately $6.3 \%$ rather than $5 \%$ precision. An increase in precision would have required reviewing an additional year's patients with bipolar disorder, and it was felt that the additional precision would not justify the effort involved or affect the outcome of the key research question.

\section{Analysis}

Data analysis was carried out using SAS (SAS Institute Inc., SAS Software, version 9.3 for Windows, Cary, NC, USA: SAS Institute Inc. [2002-2010]).

Descriptive analysis using tables and graphs to show the continuous and discrete variables (age, number of medications, psychiatric comorbidity) and categorical variables (monotherapy, polypharmacy, drug class, bipolar subtype, socio-economic status) was included.

The Chi-square $\left(\mathrm{X}^{2}\right)$ test was used to assess the association between categorical variables. The Fisher's exact test was used 
for $2 \times 2$ tables or where the requirements for the $\mathrm{X}^{2}$ test could not be met. The strength of the associations was measured by the Cramer's V and the phi coefficient - specifically in relation to psychiatric comorbidity and number of agents prescribed for the former and number of antidepressants for subtype of bipolar disorder for the latter. The following scale of interpretation was used: 0.50 and above, high/strong association; 0.30-0.49, moderate association; 0.10-0.29, weak association; below 0.10 , little if any association. The $5 \%$ significance level was used throughout, unless specified otherwise. In other words, p-values of $<0.05$ indicate significant results.

\section{RESULTS}

A total of 944 files were screened for the diagnosis of bipolar disorder for the year 2009. Of these files, 242 met the inclusion criteria and were included in the study, that is, 702 were excluded as per earlier content (Sample).
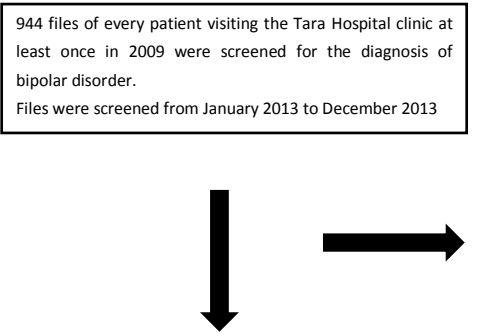

702 files were excluded from the study as they did not meet the coding and or diagnostic criteria for bipolar disorder or the clinical information in the files was insufficient for inclusion

242 patient files meeting the ICD 10 coding criteria and or DSM IV TR diagnostic criteria for bipolar disorder were included in the study

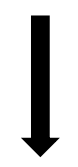

24 files for retrospective file review

Figure 1. Flowchart of the study.

\section{Descriptive Analysis of the Data Set}

Demographic Profile

\section{Age}

The patients were predominantly aged between 18 and 64 years. The mean age of the patients was 45.5 years (standard
Table 1. Demographic characteristics of patients (total $n=242$ ).

\begin{tabular}{|c|c|c|c|}
\hline Variable & Category & $\mathbf{N}$ & \% \\
\hline \multirow{4}{*}{ Age } & $18-35 y$ & 65 & 26.86 \\
\cline { 2 - 4 } & $36-50 y$ & 89 & 36.78 \\
\cline { 2 - 4 } & $51-64 y$ & 70 & 28.93 \\
\cline { 2 - 4 } & $65 y+$ & 18 & 7.44 \\
\hline \multirow{3}{*}{ Gender } & Male & 77 & 31.82 \\
\cline { 2 - 4 } & Female & 165 & 68.18 \\
\hline \multirow{4}{*}{$\begin{array}{c}\text { Hospital Fee } \\
\text { Classification }\end{array}$} & H0 & 19 & 7.85 \\
\cline { 2 - 4 } & H1 & 159 & 65.70 \\
\cline { 2 - 4 } & Private & 14 & 17.36 \\
\cline { 2 - 4 } & Others & 8 & 5.79 \\
\hline
\end{tabular}

HO hospital classification: patients on government pensions and disability grants.

H1 hospital classification: unemployed patients, students and patients with an annual income of <ZAR 36000.

$\mathrm{H} 2$ hospital classification: patients with an annual income of $\geqslant R 36000$ but $<$ R72000.

Private hospital classification: patients with an annual income of $\geqslant$ R72000.

Others: hospital classification unknown.

deviation $[\mathrm{SD}]=14.0$ years; median $=45$ years; inter quartile range $[\mathrm{IQR}]=35-55$; range $=18-84$ years; see Table 1 ).

\section{Gender}

The study group was predominantly female $(n=165 ; 68.2 \%)$ with male patients comprising $31.8 \%$ of the sample $(n=77$; see Table 1).

\section{Socio economic classification}

The hospital classification of 8 patients (3.31\%) was not available (see Table 1). Patients on government pensions and disability grants ( $\mathrm{H} 0)$ comprised $7.85 \%$ of the sample $(n=19)$. The majority of the patients $(n=159 ; 65.7 \%)$ were unemployed patients, students and patients with an annual income of <ZAR 36000 (H1) (approximately USD 3600, but because of fluctuations in currency value, this is only an approximation). Patients with an annual income of $\geq \mathrm{ZAR}$ 36000 but $<$ ZAR72000 (H2) comprised $17.3 \%$ of the sample $(\mathrm{n}=42)$. Only $5.8 \%(\mathrm{n}=14)$ were private patients (annual income $\geq$ ZAR72000). 
Table 2. Bipolar type and comorbidity profile of patients (total $n=242$ ).

\begin{tabular}{|c|c|c|c|}
\hline Variable & Category & N & $\%$ \\
\hline \multirow{2}{*}{$\begin{array}{c}\text { Bipolar disorder } \\
\text { subtype }\end{array}$} & Type I & 166 & 68.60 \\
\cline { 2 - 4 } & Type II & 56 & 23.14 \\
\cline { 2 - 4 } & NOS & 20 & 8.26 \\
\hline \multirow{2}{*}{$\begin{array}{c}\text { Psychiatric } \\
\text { comorbidity }\end{array}$} & Yes & 117 & 48.35 \\
\cline { 2 - 4 } & No & 125 & 51.65 \\
\hline
\end{tabular}

Table 3. Number of psychotropics prescribed to patients.

\begin{tabular}{|c|c|c|}
\hline Category & \multicolumn{2}{|c|}{$\begin{array}{c}\text { Number of patients per category (total } \\
\text { sample: } \mathbf{n = 2 4 2 )}\end{array}$} \\
\hline $\begin{array}{c}\text { Number of } \\
\text { psychotropics }\end{array}$ & $\mathbf{N}$ & $\%$ \\
\hline 1 & 15 & 6.20 \\
\hline 2 & 55 & 22.73 \\
\hline 3 & 84 & 34.71 \\
\hline 4 & 52 & 21.49 \\
\hline$\geqslant 5$ & 36 & 14.88 \\
\hline
\end{tabular}

\section{Diagnoses}

\section{Bipolar Disorder Subtype (DSM IV-TR)}

Type I predominated with $68.6 \%(\mathrm{n}=166)$ of patients. Type II and NOS comprised 23.1\% $(n=56)$ and $8.26 \%(n=20)$, respectively (see Table 2 ).

\section{Psychiatric Comorbidity}

Evidence of psychiatric comorbidity was recorded in $48.3 \%$ ( $\mathrm{n}=117$ ) of patients. In this study, the presence and not the specifics of the psychiatric comorbidity was recorded, as a result of some patient files lacking such details. This study included the variable 'psychiatric comorbidity' and its influence on polypharmacy and not the influence of specific psychiatric comorbidity on polypharmacy use (see Table 2).

\section{Medication Profile}

\section{Monotherapy/Polypharmacy}

As hypothesised, the majority of the sample $(93.8 \%, \mathrm{n}=227)$ were prescribed polypharmacy with only $6.2 \%(n=15)$ of the

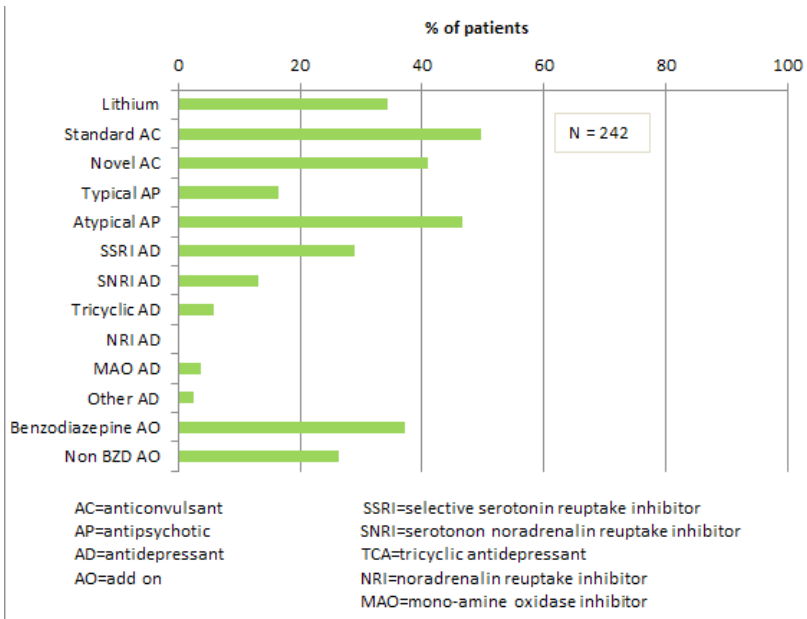

Figure 2. The percentage of patients prescribed psychotropic medications in each subclass (percentages do not sum to $100 \%$ because some patients were prescribed more than one of these medications)

patients prescribed monotherapy, that is, $22.73 \%(\mathrm{n}=55)$ of the sample were prescribed 2 psychotropic medications, $34.71 \%(\mathrm{n}=84)$ prescribed three psychotropic medications and $36.37 \%(n=88)$ prescribed four or more psychotropic medications (see Table 3).

Estimates with 95\% confidence intervals:

- Monotherapy: 6.2\% (3.5-10.0\%)

- Polypharmacy: $93.8 \%$ (90.0-96.5\%)

The mean number of psychotropic medications prescribed per patient was 3.2 as per the hypothesis $(\mathrm{SD}=1.2$; median $=$ 3 ; IQR $=2-4$; range $=1-6$ ) for the period of review.

\section{Medication}

The percentage of patients prescribed psychotropic medications in each of the medication classes is given in Figure 2. The percentages refer to the total sample unless otherwise stated as being within a specific class of medication. The majority (83.8\%) of the patients were prescribed at least one standard mood stabiliser, that is, lithium or standard anticonvulsant - noting that whilst technically lithium and standard anticonvulsants are separate classes, as noted in the study objectives, they are grouped here as mood stabilisers. As hypothesised in terms of a second agent, the prescription of antipsychotics $(61.1 \%)$ predominated, followed by add-ons (53.7\%), standard anticonvulsants (49.5\%) and antidepressants (48.7\%). Lithium was prescribed in $34.3 \%$ of patients. Sodium valproate $(37.1 \%)$ and lamotrigine $(31.8 \%)$ were the most 
popular standard and novel anticonvulsants prescribed within their classes, respectively. Within the antipsychotic class, $46.6 \%$ were prescribed atypical antipsychotics and $16.5 \%$ were prescribed typical antipsychotics. The SSRIs (28.9\%) and SNRIs (13.2\%) antidepressants were the most commonly prescribed antidepressants within their class. The benzodiazepines (39.2\%) were the most commonly prescribed add-on medication.

\section{Combinations of Medication Used}

A total of 15 patients were prescribed monotherapy (4 were prescribed lithium, 4 were prescribed a standard anticonvulsant, 5 were prescribed a novel anticonvulsant and 2 were prescribed an antipsychotic). Thus of these 15 patients, 53\% were treated with a standard mood stabiliser. No antidepressant monotherapy was prescribed in this group of patients.

The distribution of the number of classes of psychotropic medications (lithium, anticonvulsants [standard and novel], antipsychotics, antidepressants and add-on) prescribed per patient in the sample is given in Figure 3. The mean number of classes of psychotropic medication prescribed in combination was $2.8(\mathrm{SD}=0.9$; median $=3$; $\mathrm{IQR}=2-3$; range $=1-5)$.

The total number of psychotropic medication class combinations obtained was 52, involving combinations of drugs from different classes (and subclasses). Given the extent of such combinations, detailing each one would have been beyond the scope of this article, but noting the actual number underscores the uniqueness of approach that maybe required in individual patients. Of these combinations the most frequently prescribed combination was a standard anticonvulsant with an antipsychotic (9.1\%). The antipsychotics were prescribed together with standard mood stabilisers in 114 of the 242 patients (47\%). Antipsychotics were prescribed with other classes of psychotropic medication combinations in 34 of the 242 patients (14\%). Antidepressants were prescribed together with a standard mood stabiliser and/or antipsychotic in 91 of the 242 patients (38\%). Antidepressants were prescribed with a novel anticonvulsant and/or add-ons in 27 of the 242 patients (11\%).

\section{Bipolar Disorder Subtype (DSM IV-TR) and Medication Used}

The following statistically significant differences were found: The prescription of lithium was favoured for type I. The prescription of one or more standard anticonvulsants was favoured for type I. The prescription of one or more

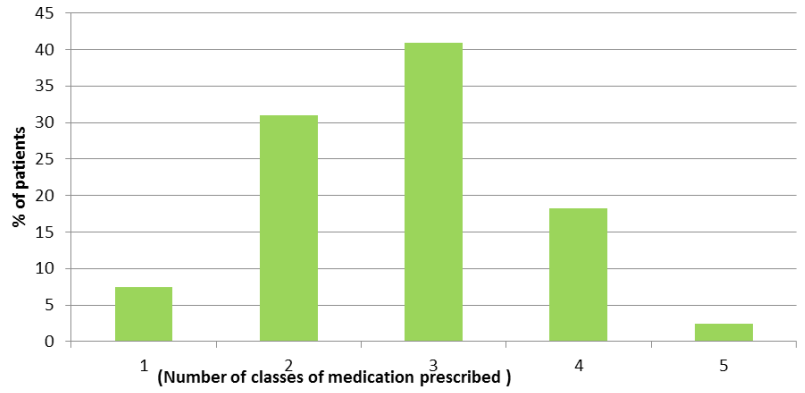

Figure 3. The distribution of the number of classes las per objectives) of psychotropic medication prescribed per patient.

novel anticonvulsants was favoured for type II and NOS. The prescription of one or more typical antipsychotics was favoured for NOS, whilst the prescription of atypical antipsychotics was favoured for type I. The prescription of one or more antidepressants was favoured for type II and NOS (Fischer's exact test; statistically significant for all prescriptions listed above with $\mathrm{p}<0.0001$ in all cases, with the strength of association for each prescription listed above being phi $=0.30,0.28,0.33,0.20,0.26,0.33,0.26$, respectively).

\section{Comorbidity and Selected Variables}

The following statistically significant differences were found:

Bipolar disorder subtype: The presence of psychiatric comorbidity was lower in type I (34.9\%) compared to type II $(75 \%)$ and NOS (85\%) (chi-square test; $\mathrm{X}^{2}[\mathrm{DF} 2]=38.6$; $\mathrm{p}<0.0001$; Cramer's V $=0.40)$.

Number of psychotropics prescribed: Of the patients with a psychiatric comorbidity, $78 \%$ were prescribed more than two medications versus $65 \%$ of those without a psychiatric comorbidity - who constituted the reference group. Those with a psychiatric comorbidity were more likely to have five or more psychotropics prescribed compared to those without psychiatric comorbidity (chi-square test; $\mathrm{X}^{2}[\mathrm{DF} 4]=10.7$; $\mathrm{p}=0.031$; Cramer's V $=0.21$ ).

Lamotrigine: Lamotrigine was prescribed more in patients with a psychiatric comorbidity (43.6\%) than in those without a psychiatric comorbidity (20.8\%) (Fisher's exact test; $\mathrm{p}<0.0001$; phi $=0.24$ ).

As describing the nature and treatment of comorbidity was not a specific objective, it was not determined which agents within the sample of polypharmacy were agents prescribed that were unrelated to bipolar disorder. 


\section{DISCUSSION}

The discussion reviews the findings of the current study and factors that are relevant to the treatment of bipolar disorder in South Africa as well as how this data accords with international literature and treatment guidelines. Specifically, it is important to note that the three stated hypotheses were confirmed.

\section{Demographic Profile}

\section{Gender}

The current study has shown a predominantly female population. This may be because females usually predominate in treated samples of patients with mood disorders (Baldessarini et al. 2007). Epidemiological studies show that bipolar disorder affects males and females equally; however, bipolar disorder II is more prominent in females (Trinh \& Forester, 2007; Sheehan 2008). It has also been reported that female patients tend to ask for help more readily than male patients and also tend to be more compliant with their medication treatment (Levine et al. 2002).

\section{Age}

The majority of the patients' age was between 18 and 64 years. This is in keeping with prevalence studies that report a one year prevalence of bipolar disorder among adults aged 65 years and older to be $0.4 \%$. This is significantly lower than in younger adults which is $1.4 \%$ (Trinh \&Forester 2007; Sheehan 2008)

\section{Socio-economic status}

Most of the patients in the current sample (65.7\%) were classified as falling into a lower socio-economic group, that is, either unemployed or earning less than ZAR36 000 a year. Tara Hospital is a government hospital and thus patients who could afford private care would likely be serviced by the private sector. Bipolar disorder is associated with high rates of unemployment (Morselli, Elgie \& Cesana 2004). This is also reflected in the current study.

\section{Diagnoses}

In the current study, bipolar disorder I was the most common diagnosis and bipolar disorder NOS was the least common diagnosis. This could be representative of the under-detection and under-treatment of bipolar spectrum disorders in psychiatric clinic settings (Merikangas et al. 2007) or could represent the nature of patients followed up having been admitted to the hospital because of symptom severity with either a manic or depressive episode. An American population study conducted between 2001 and 2003 revealed that the lifetime prevalence of bipolar disorder I was $1 \%$, bipolar disorder II was $1.1 \%$ and bipolar disorder NOS was $1.4 \%$ (Merikangas et al. 2007). Some studies estimate that bipolar disorder NOS might affect up to $6 \%$ of the general population (Merikangas et al. 2007).

Although the current study did not look at the nature of the psychiatric comorbidity, it found that almost half of the patients had a psychiatric comorbidity. The presence of psychiatric comorbidity is one of the reasons given for the use of polypharmacy in bipolar disorder (Post, 2004; Sachs et al. 2014; Hobden et al. 2013). The current study supports this association and demonstrated a statistically significant difference when comparing the number of psychotropics prescribed in the presence or absence of psychiatric comorbidity, that is, the presence of comorbidity was associated with a greater number of psychotropics prescribed. McElroy (2004) refers to bipolar disorder with comorbidity as 'complicated bipolar' and states that comorbidity is the rule not the exception in bipolar disorder. A Stanley Foundation Bipolar Treatment Outcome Network study showed that $65 \%$ of the 300 patients had at least one psychiatric comorbidity (Sagman \& Tohen 2009). Psychiatric comorbidity includes anxiety, sleep, substance and personality disorders as well as attention deficit hyperactivity disorder (American Psychiatric Association, 2000; Sagman \& Tohen 2009 ).

\section{Medication prescribing}

In the current study the standard mood stabilizers (lithium or standard anticonvulsants) were prescribed in $83.8 \%$ of patients. These results are similar to a North American study, The Systemic Treatment Enhancement Program for Bipolar Disorder study (STEP-BD), where standard mood stabilisers were prescribed in $71.9 \%$ of patients (Ghaemi, Hsu, Thase, Wisniewski, Nierenberg, Miyahara, et al. 2006). The STEP-BD is the largest treatment study conducted for bipolar disorder to date (Goldberg, Brooks, Kurita, Hoblyn, Ghaemi, Perlis et al. 2009; Ghaemi, Hsu, Thase, Wisniewski, Nierenberg, Miyahara et al. 2006). It was also conducted in academic centres. The current study revealed that standard mood stabilisers are prescribed in the majority of patients and still have an important role to play in the management of bipolar disorder. The South African and international treatment guidelines are very clear in their support of the use of these psychotropic medications and consider them to be 
first line treatment (Fountoulakis, Vieta, Sanchez-Moreno, Kaprinis, Goikolea, Kaprinis GS, 2005; Fountoulakis, Vieta, Siamouli, Valenti, Magiria, Oral, et al 2007; Colin F, 2013).

The current study found that $61.1 \%$ of patients were prescribed antipsychotics and $17 \%$ of patients were prescribed typical antipsychotics. These differences could reflect that although the patients received treatment at a tertiary institution, not all the atypical antipsychotics are readily available because of their cost and thus some require detailed motivation at consultant psychiatrist level in order to access or are not included for use on the South African Government's essential drug list because of budget constraints. The higher percentage of use of typical antipsychotics may reflect their cost effectiveness for use in a State sector outpatient setting, with depot formulations of typical antipsychotics being available for improved compliance. The higher percentage of use of antipsychotics in this South African study, in general, may reflect clinician preference, ease of use and lack of inpatient resources, thus clinicians managing severe cases in the community that require the use of antipsychotics together with standard mood stabilisers (De Las Cuevas \& Sanz 2004). The STEP-BD study found that $31 \%$ of patients were prescribed antipsychotics yet only $4 \%$ of these patients were prescribed typical antipsychotics (Ghaemi et al. 2006).

It should be noted that the South African bipolar guidelines (Colin 2013) do include the use of typical (haloperidol) and atypical antipsychotics for manic/hypomanic episodes; however, most international bipolar guidelines only include atypical antipsychotics as monotherapy or combination therapy with standard mood stabilisers (Fountoulakis et al. 2005, 2007). The South African and international bipolar treatment guidelines also suggest combination therapy with atypical antipsychotics in treating bipolar depression (Fountoulakis et al. 2005, 2007; Colin 2013). Thus the treatment guidelines recognise and support the role of antipsychotics, particularly atypical antipsychotics as monotherapy or combination therapy in the management of bipolar disorder (Fountoulakis et al. 2005, 2007; Colin 2013).

In the current study, lamotrigine $(31.8 \%)$ was the most frequently prescribed novel anticonvulsant medication. In the STEP-BD study, however, lamotrigine was prescribed in $15.4 \%$ of patients. ${ }^{[31]}$ The higher percentage of lamotrigine use in the current study may reflect clinician preference and relative ease of use because it does not require blood monitoring and is a generally safe medication to use notwithstanding recognised dermatological side effects (American Psychiatric
Association, 2010; Compton 2009). The latter consideration is particularly useful in an outpatient population. Lamotrigine is recommended to manage bipolar depression and bipolar maintenance in the treatment guidelines (Fountoulakis et al. 2005, 2007 ; Colin 2013).

The use of antidepressants in managing bipolar depression is controversial. Some studies show some benefit to the addition of an antidepressant to a standard mood stabiliser, whilst others show no positive effect (Compton 2009; Ghaemi, Hsu, Goodwin 2003). Bipolar treatment guidelines suggest cautious use of antidepressants: use in combination with an anti-manic medication (standard mood stabilizer, antipsychotic) and careful/selective choice of an SSRI in order to prevent a manic switch or rapid cycling (Fountoulakis et al. 2005, 2007; Colin 2013). The prescribing of antidepressants to patients in the STEP-BD study (Ghaemi et al. 2006) and the current study was noted to be between $40 \%$ and $50 \%$. The antidepressant use in both studies is high, given the concerns around treating patients with bipolar disorder in the depressed phase with antidepressants. Such prescribing can, however, be justified, given that patients are treated in specialist, academic settings potentially implying both complexity as well as an understanding of the risks and benefits. That 15$20 \%$ of patients with bipolar disorder relapse into depression after discontinuation of antidepressant suggests the need for antidepressants (Ghaemi, Hsu\& Goodwin 2003).

In the current study it was found that the SSRIs were the most common antidepressant prescribed $(28.9 \%)$ and that a minority of patients (11\%) were prescribed an antidepressant without an anti-manic medication. The predominant use of SSRIs was also observed in the STEP-BD study (21.6\%) (Ghaemi et al. 2006). These results reflect the clinicians' compliance with treatment guidelines (Fountoulakis et al. 2005, 2007; Colin 2013).

In the current study, patients with bipolar I disorder were prescribed standard mood stabilisers and atypical antipsychotics more often than the other bipolar types. The patients with bipolar I disorder experience mania and clinical trials and bipolar treatment guidelines advocate the use of these medications for manic episodes and maintenance of bipolar I disorder (Fountoulakis et al. 2005, 2007; Colin 2013). The favouring of antipsychotics, in particular the typical agents, in the bipolar NOS group may suggest the comorbidity of Cluster B (borderline, narcissistic and anti-social) personality disorders (American Psychiatric Association, 2000) with bipolar disorder. 
Prevalence studies show that patients with bipolar disorder have a significantly higher prevalence of personality disorder than the general population (Fan \& Hassel 2008). Ehret demonstrated in a bipolar study that $53.4 \%$ had a comorbid personality disorder and most had borderline personality disorder (Compton M, 2009). The antipsychotics are used to treat mood instability, aggression and impulsivity in Cluster B personalities (American Psychiatric Association, 2010). The researcher's personal experience as a consultant psychiatrist at Tara Hospital outpatient's department highlighted the utility of flupenthixol depot (typical antipsychotic) usage in low doses because of its effectiveness, low side effects and low cost.

\section{Comorbidity and bipolar disorder}

The current study did not address the spectrum of comorbidity and association with bipolar type, but it did demonstrate that bipolar II and NOS have a higher presence of comorbidity. Bipolar II disorder often has more depressive episodes than the other types of bipolar disorder, whilst bipolar disorder, especially bipolar II, and borderline personality disorder often co-occur. ${ }^{[31,38]}$ This could then explain the preference of antidepressants and novel anticonvulsants in the current study's bipolar II and NOS groups. Lamotrigine and antidepressants are recommended to manage depression, mood instability and impulsivity in borderline personality disorders and depressive episodes and maintenance treatment in bipolar disorder (Fountoulakis et al. 2005, 2007; Colin 2013; American Psychiatric Association 2010). The current study revealed that lamotrigine was favoured in those patients with comorbidity.

\section{Monotherapy / Polypharmacy for bipolar disorder}

The current study has shown that the use of polypharmacy was practiced in the majority of patients (93.8\%), with 'majority' defined by the current study as $>50 \%$ of patients, which is in keeping with international literature that includes data from Asian studies (Goldberg et al. 2009; Tohen et al. 2002; Post 2004; Gever 2010; Ventimiglia, Kalali \& McIntyre 2009; Baldessarini et al. 2007; Post et al. 2010; Hooshmand et al. 2014; De Las Cuevas \& Sanz 2004). The current study, however, shows a much higher percentage of polypharmacy use, which from international literature was estimated to be between 50\% and 60\% (Goldberg et al. 2009; Tohen et al. 2002; Post 2004; Gever 2010; Ventimiglia, Kalali \& McIntyre 2009; Baldessarini et al.2007; Post et al. 2010; Hooshmand et al. 2014; De Las Cuevas \& Sanz 2004). This may once again reflect the severity of the patients whom clinicians are needing to manage as outpatients because of a lack of inpatient resources or the nature of patients initially referred for admission who were subsequently being followed up (De Las Cuevas \& Sanz 2004).

The patients were prescribed a mean number of 3.2 psychotropic medications. This was also in keeping with international studies that showed the average number of medications prescribed to be 3.3, 2.98 and 2.6 (Post et al. 2010; Hooshmand et al. 2014 ; Weinstock et al. 2014). The STEPBD study showed that $40 \%$ of the patients were prescribed three or more concurrent psychotropic medications and $18 \%$ of patients received four or more psychotropic medications (complex polypharmacy). The use of complex polypharmacy was found in $36.3 \%$ of patients in the current study. This may once again, as mentioned above, highlight the complexity and severity of patients being managed as outpatients and unmet inpatient needs (De Las Cuevas \& Sanz 2004).

The current study highlights and is supported by many studies that bipolar disorder is more commonly treated with polypharmacy than monotherapy (Goldberg et al. 2009; Post 2004; Ventimiglia, Kalali \& McIntyre 2009; Baldessarini et al. 2007; Post et al. 2010; Hooshmand et al. 2014; Levine et al. 2000; Weinstock et al. 2014). The current study also demonstrates that patients being treated within a specialist academic psychiatric setting are more likely to be prescribed polypharmacy. This finding was congruent with the STEP-BD study (Goldberg et al. 2009) and with Ventimiglia's comment: 'the majority of individuals receiving care in tertiary and specialty centers will require a polytherapeutic regimen' (Ventimiglia, Kalali \& McIntyre 2009 ). Nichol et al (1995) looked at factors predicting the use of multiple psychotropic medications and found that patients consulting psychiatric specialists are much more likely to be prescribed polypharmacy than those who consulted general practitioners. The patients consulting psychiatrists were six times more likely to receive multiple psychotropic medications. Nichol et al. (1995) also found that manic patients were four times more likely to be treated with polypharmacy. These findings suggest that complicated mental health problems require polypharmacy and are more likely to be supervised by specialist psychiatrists than general practitioners/primary care physicians (Goldberg et al. 2009; Ventimiglia, Kalali \& McIntyre 2009; Nichol, Stimmel \& Lange 1995).

\section{Combinations of psychotropic medication}

The majority of patients were prescribed more than two classes of psychotropic medication (mean $=2.8$ ). There was no combination of psychotropic medication classes prescribed 
that predominated, and 52 combinations were shown. The most common combination is a standard anticonvulsant and an antipsychotic, although this was only prescribed in $9.1 \%$ of patients. These findings are in keeping with two other studies (Bauer et al. 2013; Rossenbaum \& Covino 2007) that looked at prescribing patterns in bipolar disorder. Bauer et al. (2013) showed 52 combinations and Rossenbaum and Covino (2007) showed 38 combinations of classes of psychotropic medication prescribed. The current study did, however, find that $47 \%$ of the combinations did include at least a standard mood stabiliser and an antipsychotic. This finding is consistent with the data that suggest the use of a standard mood stabiliser together with an antipsychotic may be more effective than either class of medication on their own (Blanco et al. 2002).

Many patients with bipolar disorder, however, do not respond to combinations of treatments consistent with evidencebased guidelines (Sagman \& Tohen 2009). The literature to date has not adequately addressed the efficacy of combination treatment involving more than two psychotropic medications to treat patients with bipolar disorder (Sagman \& Tohen 2009). Sachs raises concerns that this leads to personalised and individualised care that is uncontrolled and does not meet the standards of evidence-based medicine (Sagman \& Tohen 2009). Gelenberg and Pies (2003) advocate that clinicians should match a particular patient with the optimal regimen or customise treatment to each patient's needs whilst practicing effective and safe prescribing. They encourage clinicians to look at previous response to treatment, polarity of an episode, psychiatric and medical comorbidity and drug interactions (Gelenberg \& Pies 2003). Thus these two author's views could account for the large variability observed in combination treatment for patients with bipolar disorder.

\section{LIMITATIONS}

The study is a retrospective file review and relies on the information documented in the files. This information may not always be reliable or recorded in sufficient detail. The study also presumes that mental health care professionals have sufficient knowledge of the DSM IV TR (American Psychiatric Association 2000) and ICD 10 (World Health Organization 1992) classifications and have used it correctly when making diagnoses, undertaking assessments and recording clinical information in the file. When it came to the data collection and analysis, however, the ICD 10 codes (World Health Organization 1992) for bipolar disorder were not used as they were not consistently or correctly recorded. The researcher relied on the DSM IV TR (American Psychiatric Association 2000) diagnosis recorded together with the clinical notes in the file documenting DSM IV TR diagnostic criteria (American Psychiatric Association 2000) to provide and record the bipolar subtype. The psychiatric comorbidity was not documented in detail because the clinical notes did not adequately document this.

The monotherapy group was small, and whilst proving one of the hypotheses, the ability to make inferences for this group was limited. As the study was cross sectional, the information gathered pertains to a particular period, that is, patients seen at the clinic in 2009 with their last prescription for 2009. The data cannot be, therefore, generalised beyond this environment/period. Notwithstanding the timing of the study, it should be noted that no similar such data from South Africa has been published, either prior or subsequent at the time of writing.

The current study did not consider a specific bipolar disorder episode but rather considered a general approach to prescribing in bipolar disorder. The study made an assumption that the majority of patients would be in the maintenance phase of bipolar disorder given that the study population were outpatients. The study could not exclude that some patients may have been in an acute relapsing/recurring phase but that they could be managed in an outpatient setting.

\section{CONCLUSION}

The current study is South African based. The results from the study are congruent with independent conclusions of numerous international studies highlighted throughout the discussion. The conclusion is that polypharmacy use in bipolar disorder is the norm and where polypharmacy is prescribed, there is a diverse spectrum of psychotropic medication combinations used to treat bipolar disorder (Gever 2010; De Las Cuevas \& Sanz 2004; Bauer et al. 2013). This may be a consequence of the complexity of bipolar disorder, which includes recurrent and various episodes, psychiatric comorbidity, the increasing trend by clinicians to treat symptoms and inadequate guidelines and studies with respect to recommended combinations - in particular when more than two psychotropic medications are involved (Gever 2010; De Las Cuevas \& Sanz 2004; Bauer et al. 2013).

The study does highlight, however, that South African clinician prescribing in a specialised out-patient academic setting is congruent with the medication choices suggested by the international and local bipolar treatment guidelines 
(Fountoulakis et al. 2005, 2007; Colin 2013). From the current study, the many combinations of psychotropic medication prescribed may be due to clinician personal preference as well as the need to individualise treatment according to the needs of each patient, which include clinical, social, economic and pharmacological aspects (De Las Cuevas \& Sanz 2004; Bauer et al. 2013). The evaluation and validity or otherwise of this hypothesis would be an interesting basis for further study.

\section{ACKNOWLEDGEMENTS}

A special thanks to Tara Hospital, the staff at the Tara Hospital outpatient clinic and the statistician Dr Petra Gaylard.

\section{CONFLICT OF INTEREST STATEMENT}

The authors report no conflict of interest related to this manuscript.

\section{FUNDING}

This study required no external funding.

\section{ETHICS APPROVAL}

The protocol for this study was approved by the Human Research Ethics Committee at the University of the Witwatersrand (protocol number M120838) on 31 August 2012. The permission was also obtained from the Chief Executive Officer of TARA Hospital to conduct the study at the hospital.

\section{INFORMED CONSENT}

No informed consent was obtained for this study because the study was a chart review.

\section{AUTHORS' CONTRIBUTIONS}

Dr Eleanor Holzapfel was responsible for the study design, data collection, writing and revision of the article. Prof Christopher Szabo was responsible for the study design, writing and revision of the article.

\section{REFERENCES}

Alda, M \& Yatham, LN 2009, 'Is monotherapy as good as polypharmacy in long- term treatment of bipolar disorder?', The Canadian Journal of Psychiatry, vol.54, no. 11, pp.719-725.

American Psychiatric Association 2000. Diagnostic and statistical manual of mental disorders (Revised 4th ed.), American Psychiatric Association, Washington, DC.

American Psychiatric Association, 2010, 'Practice guidelines for the treatment of patients with borderline personality disorder', Psychiatry online http://www.psychiatryonline.org/pb/assets/raw/ sitewide/practice guidelines/bpd.

Baldessarini, R, Leahy, L, Arcona, S, Gause, D, Zhang, W \& Hennen, J 2007, 'Patterns of psychotropic drug prescriptions for U.S. patients with diagnoses of bipolar disorders', Psychiatric Services, vol. 58, pp.85-91. doi:10.1176/ps.2007.58.1.85

Bauer, M, Glenn, T, Alda, M, Sagduyu, K, Marsh, W, Grof, P, Munoz, R, Severus E, Ritter, P \& Whybrow, PC 2013, 'Drug treatment patterns in bipolar disorder: analysis of long term self reported data', International Journal of Bipolar Disorders, vol.1, no.5 doi: 10.1186/2194-7511-1-5
Blanco, B, Gonzalo, L, Olfson, M, Marcus, S \& Pincus H 2002, 'Trends in the treatment of bipolar disorder by outpatient psychiatrists', The American Journal of Psychiatry, vol. 159, no.6, pp.1005-1010.

Colin, F 2013, 'Bipolar disorder-The South African society of psychiatrists treatment guidelines for psychiatric disorders', South African Journal of Psychiatry, vol. 19, no. 3, pp.164-170.

Compton, M 2009, 'Recent research in bipolar disorder: treatment', Medscape Psychiatry and Mental Health Available from: http:// www.medscape.org/viewarticle/704536

De Las Cuevas, C \& Sanz, E 2004, 'Polypharmacy in psychiatric practice in the Canary Islands', BMC Psychiatry, vol. 4, no.18 https://doi.org/10.1186/1471-244X-4-18

Fan, A, \& Hassell, J 2008, 'Bipolar disorder and comorbid personality psychopathology; a review of the literature', Journal of Clinical Psychiatry, vol.69, no.11. pp.1794-1803.

Fiedorowicz, J \& Black, D 2010, 'Borderline, bipolar or both? Frame your diagnosis on patient history'. Current Psychiatry, vol.9, 
no.1, pp.21-30 Available from: http://www.imn.gcnpublishing. com/fileadmin/cp_archive/pdf/0901/0901CP_article1.

Fountoulakis, KN, Vieta, E, Sanchez-Moreno, J, Kaprinis, SG, Goikolea, JM \& Kaprinis, GS 2005 'Treatment guidelines for bipolar: a critical review', Journal of Affective Disorders, vol. 86, no.1, pp.1-10.

Fountoulakis, KN, Vieta, E, Siamouli, M, Valenti, M, Magiria, S, Oral, T, Fresno, D, Giannakopoulos, P \& Kaprinis, GS 2007, 'Treatment of bipolar disorder: a complex treatment for a multifaceted disorder', Annals of General Psychiatry, vol. 6, no. 27, pp.1-12. doi:10.1186/1744-859X-6-27.

Frye, MA, Ketter, TA, Leverich, GS, Huggins, T, Lantz, C, Denicoff KD \& Post, RM 2000, 'The increasing use of polypharmacotherapy for refractory mood disorders: 22 years of study', Journal of Clinical Psychiatry, vol. 61, no.1 , pp. 9-15.

Frye, MA 2011, 'Bipolar disorder- A focus on depression', New England Journal of Medicine, vol. 364, pp. 51-59. doi:10.1056/ NEJMcp1000402.

Gelenberg, AJ \& Pies, R 2003, 'Matching the bipolar patient and the mood stabilizer', Annals of Clinical Psychiatry, vol. 15, no.3-4, pp.203-216.

Gever, J, 2010, 'Psychiatric polypharmacy continues to grow' Medpage today http://www.medpagetoday.com/psychiatry/ generalpsychiatry/17785.

Ghaemi, S, Hsu, D, Goodwin, F 2003, Antidepressants in bipolar disorder; the case for caution', Bipolar Disorders, vol. 5, no.6, pp. 421-433. doi:10.1046/j.1399-5618.2003.00074.x.

Ghaemi, SN, Hsu, DJ, Thase, ME, Wisniewski, SR, Nierenberg, AA, Miyahara, S \& Sachs G 2006, 'Pharmacological treatment patterns at study entry for the first 500 STEP-BD participants', Psychiatric Services, vol.57, no. 5, pp. 660-665. https://ps.psychiatryonline. org/doi/full/10.1176/ps.2006.57.5.660

Goldberg, JF, Brooks, JO, Kurita, K, Hoblyn, JC, Ghaemi, SN, Perlis, RH, Miklowitz, DJ, Ketter, TA, Sachs, GS, \& Thase, ME 2009, 'Depressive illness burden associated with complex polypharmacy in patients with bipolar disorder: findings from the STEP-BD', Journal of Clinical Psychiatry, vol. 70 no. 2, pp.155-162.

Goodwin, G, Fleischhacker, W, Arango, C, Baumann, P, Davidson, M, de Hert, M, Falkai, P, Kapur, S, Leucht, S, Licht, R, Naber, D, O'Keane, V, Papakostas, G, Vieta, E \& Zohar J 2009, 'Advantages and disadvantages of combination treatment with antipsychotics',
European Neuropsychopharmacology vol. 19, pp. 520-532. doi: 10.1016/j.euroneuro.2009.04.003

Hobden, K, Preethy, S, LeRoy, B, \& Lindsay, D 2013, 'An empirical examination of the prevalence and predictors of polypharmacy in individuals with dual diagnosis', International Journal of Disability, Community and Rehabilitation, vol. 12; no.1 http://www.ijdcr.ca/ VOL12_01/articles/hobden.shtml

Hooshmand, F, Miller, S, Dore, J, Wang, P, Hill, S, Portillo, N \& Ketter, TA 2014, 'Trends in pharmacotherapy in patients referred to a bipolar speciality clinic, 2000-2011', Journal of Affective Disorders , vol. 155, no. 2. Pp. 283-287. doi:10.1016/j.jad.2013.10.054.

Hung, GC, Yang, SY, Chen, Y \& Lin SK 2014 'Psychotropic polypharmacy for the treatment of bipolar disorder in Taiwan', Psychiatric services, vol. 65, no. 1, pp.125-128. doi: 10.1176/appi.ps.201200529.

Jarema, M, Hotujac, L, Oral, ET, Rybakowski, J, Sartorius, N \& Svestka, J 2005, 'Treatment of bipolar disorders with second generation antipsychotic medications', Neuroendocrinology Letters, vol. 26(suppl 1), pp. 1-79.

Kukreja, S, Kalra, K, Shah, S \& Shrivastava A 2013 'Polypharmacy in psychiatry: a review', Mens Sana Monographs vol. 11, no.1, pp. 82-99. doi:10.4103/0973-1229.104497.

Levine, J, Chengappa, K, Brar, J, Gershon, S, Yablonsky, E, Stapf, D \& Kupfer, DJ 2000 'Psychotropic drug prescription patterns among patients with bipolar I disorder', Bipolar Disorders, vol. 2, no. 2, pp.120-130. doi:10.1034/j.1399-5618.2000.020205.x.

Mc Elroy, S 2004, 'Diagnosing and treating comorbid (complicated bipolar disorder)', Journal of Clinical Psychiatry, vol.65S, no.15, pp. 35-44.

Merikangas, K, Akiskal, H, Angst, J, Greenberg, P, Hirschfield, R, Petukhowa, M \& Kessler, RC 2007, 'Lifetime and 12-month prevalence of bipolar spectrum disorder in the national comorbidity survey replication', Archives of General Psychiatry, vol. 64, no. 5, pp.543-552. doi: 10.1001/archpsyc.64.5.543

Morselli, P, Elgie, R \& Cesana, B 2004, 'GAMIAN-Europe/BEAM survey II: cross-national analysis of unemployment, family history, treatment satisfaction and impact of bipolar disorder on life style', Bipolar Disorders, vol. 6 no. 6, pp. 487-497 DOI: 10.1111/j.13995618.2004.00160.x

Nichol, M, Stimmel, G \& Lange, S 1995 'Factors predicting the use of multiple psychotropic medications', Journal of Clinical Psychiatry, vol. 56, no. 2, pp. 60-62. 
Post, RM, 2004, 'Monotherapy vs. polypharmacy', Medscape Psychiatry \& Mental Health, vol.9, no.1, pp.1-5 Available from: http://cme.medscape.com/viewarticle/468952. [accessed 2010 Jun. 2] .

Post, R, Altshuler, L, Frye, M, Suppes, T, Keck, P, McElroy, S, Leverich, GS, Luckenbaugh, DA, Rowe, M, Pizzarello, S, Kupka, RW, Grunze, H \& Nolen WA 2010, 'Complexity of pharmacologic treatment required for sustained improvement in outpatients with bipolar disorder', Journal of Clinical Psychiatry, vol. 71, no. 9, pp. 1176-1186. doi:10.4088/JCP.08m04811yel.

Rossenbaum, J, \& Covino, J 2007, 'Observations on the treatment of bipolar disorder: pharmacotherapy for bipolar depression', Medscape Psychiatry, Available from: http://www.medscape.org/ viewarticle/563043.

Sachs, G, Peters, A, Sylvia, A \& Grunze, H 2014, 'Polypharmacy and bipolar disorder: what's personality got to do with it?', International Journal of Neuropsychopharmacology, vol.17, pp.1053-1061. doi:10.1017/S1461145713000953.

Sagman, D \& Tohen, N 2009, 'Comorbidity in bipolar disorder', Psychiatric Times Available from: http//www.psychiatrictimes. com/bipolar-disorder/comorbidity-bipolar-disorder

Sheehan, J 2008. 'Bipolar disorders in seniors', Bipolar Disorder Center Available from: http://www.everydayhealth.com/bipolardisorder/bipolar-disorder-in-seniors.aspx.
Solomon, D, Keitner, G, Ryan, C \& Miller, T 1996 'Polypharmacy in bipolar I disorder' Psychopharmacology Bulletin, vol. 32, no.4, pp. 579-587.

Tohen, M, Chengappa, K, Suppes, T, Zarate, CA, Calabrese, JR, Bowden, CL, Sachs, GS, Kupfer, DJ, Baker, RW, Risser, RC, Keeter, EL, Feldman, PD, Tollefson, GD \& Breier A 2002, 'Efficacy of olanzapine in combination with valproate or lithium in the treatment of mania in patients partially nonresponsive to valproate or lithium monotherapy' Archives of General Psychiatry, vol. 59, pp. 62-69. doi:10.1001/archpsyc.59.1.62.

Trinh, N \& Forester, B 2007 'Bipolar disorder in the elderly: differential diagnosis and treatment', Psychiatric Times Available from: http://wwwpsychiatrictimes.com/bipolar/bipolar-disorderelderly.

Ventimiglia, J, Kalali, AH, \& Mclntyre, RS 2009, 'Treatment of bipolar disorder', Psychiatry, vol. 6, no. 10, pp. 12-14.

Weinstock, L, Gaudiano, B, Epstein-Lubow, G, Tezanos, K, CelisDehoyos, C \& Miller, I 2014, 'Medication burden in bipolar disorder: a chart review of patients at psychiatric hospital admission', Psychiatric Residence, vol. 216, no.1, pp.24-30.

World Health Organization 1992. International classification of disease and related health problems (Revised 10th ed.), World Health Organization, Geneva. 
